\title{
Kontaktallergie auf FFP2-Maske in der COVID-19-Pandemie
}

\section{Case report of contact allergy to filtering facepiece class $\mathbf{2}$ mask of a medical worker during Covid-19 pandemic}

\section{Einführung}

Am 11. Februar 2020 wurde die klinische Symptomatik der Infektion mit den neuartigen humanpathologischen Coronaviren (Severe acute respiratory syndrome-Coronavirus 2: SARS-CoV-2) als „CoronavirusKrankheit 2019“ (COVID-19) benannt [1]. Die globale Ausweitung der SARS-CoV-2Pandemie und die mögliche Schwere des klinischen Verlaufs stellen die Gesundheitssysteme weltweit vor große Herausforderungen. Das Robert-Koch-Institut (RKI) empfiehlt in Übereinstimmung mit dem Europäischen Zentrum für Prävention und Kontrolle von Krankheiten (ECDC) und der WHO in der aktuellen Pandemiesituation präventive Maßnahmen, wie z. B. die Einhaltung eines Abstands von 1,5 bis 2 Metern zwischen den Menschen, die Förderung der Einhaltung allgemeiner Hygienemaßnahmen wie regelmäßige Händedesinfektion/ regelmäßiges Händewaschen für mindestens 30 Sekunden, die Vermeidung von Berührungen der Schleimhäute mit den Händen, der Minimierung sozialer Kontakte und Beschränkung persönlicher Patientenkontakte auf das absolut notwendige Maß, die regelmäßige Desinfektion von Oberflächen, insbesondere von Türgriffen, und insbesondere auch das Tragen persönlicher Schutzkleidung wie Mund-Nasen-Masken (MNM). Bei den MNM sind im Wesentlichen zu unterscheiden 1.) „Community-Masken“ (Behelfs-Mund-Nasen-Masken aus handelsüblichen Stoffen), 2.) medizinische Gesichtsmasken und 3.) filtrierende Halbmasken. Nur für 2.) und 3.) existieren einschlägige gesetzliche Vorgaben und technische Normen, sodass von einer Schutzmaske im eigentlichen Sinn mit ausgelobter Schutzwirkung ausgegangen werden kann. Filtrierende Halbmasken (FFP1-, FFP2-, FFP3-Masken) werden in Deutschland gemäß der Norm DIN EN 149:2001-10 geprüft. Nachdem zu Beginn der COVID-19-Pandemie sowohl medizinische Gesichtsmasken als auch filtrierende Halbmasken der Klassifizierung FFP1 bis FFP3 kaum erhältlich waren, sind inzwischen v. a. FFP2-Masken auch über den medizinischen Bereich hinaus weit verbreitet.

\section{Fallbeschreibung}

Wir berichten über eine 41-jährige Patientin, die als medizinische Fachangestellte in einer Arztpraxis arbeitet. Im Rahmen der Pandemiesituation wurden in vielen Bundesländern medizinische Gesichtsmasken und filtrierende Halbmasken zentral beschafft und über die Kassenärztlichen Vereinigungen an die Praxen verteilt. Somit bestand kein Einfluss auf die Art und den Hersteller der MNM und die Produkte wechselten „je nach Verfügbarkeit“ häufig.

Nachdem die Patientin mehrere FFP2Masken auch bei längerer Tragezeit (Tagschicht) gut vertragen hatte, reagierte sie am vierten Tag der Nutzung des neuen FFP2-Maskentyps eines chinesischen Herstellers mit massiv juckenden, geröteten, nässenden und später schuppenden Hautausschlägen. Auch nach ausgiebiger Nachforschung konnte für das betreffende Produkt keine CE/NE-Kennzeichnung gefunden werden.

\section{Diskussion}

Filtrierende Halbmasken der Klassifizierung FFP2 sind aktuell im medizinischen Bereich die wohl am häufigsten verwendeten Gegenstände der persönlichen Schutzausrüstung (PSA). Sie haben die Zweckbestimmung, den Träger der Maske vor Partikeln, Tröpfchen und Aerosolen zu schützen, können aber auch im Falle einer Infektion des Trägers die Verbreitung von Aerosol-getragenen Viren verhindern. Letzteres gilt aber abhängig vom Design der filtrierenden Halbmasken nur für Masken ohne Ausatemventil, die sowohl die eingeatmete Luft als auch die Ausatemluft filtern und daher sowohl einen Eigenschutz als auch einen Fremdschutz bieten. Masken mit Ausatemventil hingegen filtern nur die eingeatmete Luft und sind daher nicht für den Fremdschutz ausge- legt. Um FFP-Masken rechtmäßig in Deutschland und Europa in den Verkehr zu bringen, muss für diese ein Konformitätsbewertungsverfahren gemäß PSA-Verordnung (EU) 2016/425 durchgeführt werden, nach dessen Abschluss sie vom Hersteller mit einem CE-Kennzeichen versehen werden. Die Masken müssen dazu regulär die Anforderungen der Norm DIN EN 149:2001-10 erfüllen. Weitere Informationen zum rechtmäßigen Inverkehrbringen von PSA in Deutschland sind auf der Homepage der Bundesanstalt für Arbeitsschutz und Arbeitsmedizin (BAuA) zu finden [2] (Zugriff 23.05.2020). Abweichend von der o.g. Einordnung können FFP-Masken in Ausnahmefällen auch gem. $\S 2$ Abs. 4a des Medizinproduktegesetzes (MPG) als Medizinprodukte im Verkehr sein, wenn sie nicht über ein Ausatemventil verfügen (die Luft also beim Ein- und Ausatmen gefiltert wird), vom Hersteller mit medizinischer Zweckbestimmung gemäß § 3 Abs. 1 MPG in Verkehr gebracht werden und das BfArM in der aktuellen Bedarfssituation auf Basis einschlägiger Sicherheits- und Leistungsnachweise eine entsprechende Sonderzulassung nach §11 Abs. 1 MPG erteilt hat. Zudem wurden Sonderregelungen geschaffen für die aktuelle Pandemiesituation [3] (Zugriff 23.05.2020). Demnach gelten „...zur Bewältigung der aktuellen Krisenlage bezüglich der Eindämmung von COVID-19 ... medizinische Gesichtsmasken und FFPMasken, die in den Vereinigten Staaten von Amerika, Kanada, Australien oder Japan verkehrsfähig sind, ggf. auch in Deutschland als verkehrsfähig, auch wenn diese keine CE/NE-Kennzeichnung tragen“.

Bei der betroffenen Patientin wurde durch die FFP2-Maske ein Kontaktekzem (Syn. Kontaktdermatitis) als entzündliche, nichtinfektiöse Intoleranzreaktion der Haut ausgelöst [4].

Kontaktekzemen liegt eine immunologisch, chemisch oder physikalisch wirkende Noxe zugrunde. Als Auslöser kommen überwiegend Allergene und irritative Substanzen/Einwirkungen infrage. Die klini- 
schen Symptome sind sehr variabel und werden von verschiedenen Faktoren beeinflusst. Häufig kommt es zu Rötungen, Schuppungen, Juckreiz und Bläschen. Teilweise treten Schwellungen, nässende Läsionen, Verhornungen und Hautrisse auf. Kontaktekzeme sind häufige Erkrankungen und können insbesondere bei chronischem Verlauf die Betroffenen sehr belasten.

Grundsätzlich kommt differenzialdiagnostisch bei der Patientin ursächlich eine physikalisch-irritative neben einer chemischen oder allergisch-immunologischen Noxe infrage [4]. Das irritative Kontaktekzem (Syn. toxisches Kontaktekzem) ist eine unspezifische, auf das Kontaktareal begrenzte Entzündungsreaktion der Haut und Schleimhäute durch Exposition gegenüber hautreizenden Substanzen/ Einwirkungen (z. B. Feuchtigkeit, Säuren, Laugen, mechanische Belastungen). Zu unterscheiden sind das akute irritative Kontaktekzem nach Exposition gegenüber einer stärker irritativ wirkenden Noxe (z. B. nach Kontakt mit Säure) und das chronische irritative Kontaktekzem (Syn. kumulativ-subtoxisches Kontaktekzem), welches durch wiederholten Kontakt zu einer schwächeren Noxe entsteht. Ein spezieller Test steht für die Diagnose des irritativen Kontaktekzems nicht zur Verfügung; daher sollte durch eine zielgerichtete allergologische Diagnostik eine immunologische Ursache des Kontaktekzems gesucht bzw. ausgeschlossen werden.

Kontaktallergien basieren auf einer T-Zell-vermittelten spezifischen Reaktion (Typ-IV-/Spättyp-Sensibilisierung) gegenüber reaktiven Substanzen, die meist als Haptene erst nach Bindung an Proteine in der Haut zu einem Allergen werden [4]. Es handelt sich somit meist um eine Allergie gegenüber kleinmolekularen Substanzen. Bekannte Kontaktallergene sind unter anderem Metalle (z. B. Nickel), Konservierungsmittel, Duft- und Farbstoffe, Gummi-Inhaltsstoffe, Bestandteile von Harzen und Klebern sowie Kunststoff-Monomere (z. B. Epoxidharz, Acrylate und Desinfektionsmittel). Das allergische Kontaktekzem ist die klinische Manifestation einer Kontaktallergie. Bei Betroffenen mit klinisch relevanter Sensibilisierung kommt es nach Exposition gegenüber einem Kontaktallergen meist zeitversetzt (ca. 13 Tage später) zu entzündlichen Hautver-

- Tab. 1 Standard-Testreihe der Deutschen Kontaktallergie-Gruppe (DKG).

\begin{tabular}{|c|c|c|c|c|}
\hline 1 & Kaliumdichromat & 0,5 & $\%$ & Vas. \\
\hline 2 & Thiuram-Mix & 1 & $\%$ & Vas. \\
\hline 3 & Kobalt (II)-chlorid, $6 * \mathrm{H} 20$ & 1 & $\%$ & Vas. \\
\hline 4 & Perubalsam & 25 & $\%$ & Vas. \\
\hline 5 & Kolophonium & 20 & $\%$ & Vas. \\
\hline 6 & N-Isopropyl-N’-phenyl-p-phenylendiamin & 0,1 & $\%$ & Vas. \\
\hline 7 & Wollwachsalkohole & 30 & $\%$ & Vas. \\
\hline 8 & Mercapto-Mix ohne MBT (nur CBS, MBTS, MOR) & 1 & $\%$ & Vas. \\
\hline 9 & Epoxidharz & 1 & $\%$ & Vas. \\
\hline 10 & Methylisothiazolinon & 0,05 & $\%$ & Aqu. \\
\hline 11 & Nickel (II)-sulfat, 6*H20 & 5 & $\%$ & Vas. \\
\hline 12 & Formaldehyd & 1 & $\%$ & Aqu. \\
\hline 13 & Duftstoff-Mix & 8 & $\%$ & Vas. \\
\hline 14 & Terpentin & 10 & $\%$ & Vas. \\
\hline 15 & Propolis & 10 & $\%$ & Vas. \\
\hline 16 & 2-Hydroxyethylmethacrylat (HEMA) & 1 & $\%$ & Vas. \\
\hline 17 & (Chlor)-Methylisothiazolinon (MCI/MI) & 100 & $\mathrm{ppm}$ & Aqu. \\
\hline 18 & Sandelholzöl & 10 & $\%$ & Vas. \\
\hline 19 & Compositae Mix II & 5 & $\%$ & Vas. \\
\hline 20 & Mercaptobenzothiazol & 2 & $\%$ & Vas. \\
\hline 21 & HICC (Lyral) & 5 & $\%$ & Vas. \\
\hline 22 & lodpropinylbutylcarbamat & 0,2 & $\%$ & Vas. \\
\hline 23 & Duftstoff-Mix II & 14 & $\%$ & Vas. \\
\hline 24 & Sorbitansesquioleat & 20 & $\%$ & Vas. \\
\hline 25 & Ylang-ylang (I + II) ÖI & 10 & $\%$ & Vas. \\
\hline 26 & Jasmin absolut & 5 & $\%$ & Vas. \\
\hline 27 & Natriumlaurylsulfat (SLS) & 0,25 & $\%$ & Aqu. \\
\hline
\end{tabular}

änderungen in den Kontaktarealen (selten darüber hinausgehend). Therapeutisch wichtig bei einer Kontaktallergie ist in erster Linie die Allergenmeidung. Der diagnostischen Abklärung und Ermittlung des auslösenden Kontaktallergens kommt daher eine besondere Bedeutung zu. Im Mittelpunkt steht dabei der Epikutantest (Synonym: Patch-Test), mit dem eine Sensibilisierung gegenüber einem Kontaktallergen nachgewiesen oder ausgeschlossen werden kann [4].

Bei der Patientin wurde ursächlich eine Kontaktallergie auf Formaldehyd nachgewiesen. Aldehyde wie Formaldehyd und Glutaraldehyd werden im medizinischen Bereich breit eingesetzt, u. a. als Flächenund Instrumentendesinfektionsmittel, aber auch in der Herstellung von Schutzkleidung und sonstiger PSA, und stellen daher Kontaktallergene mit erheblicher berufsdermatologischer Relevanz in medizinischen Berufen dar [5].

Eine Testung ist mit der DKG-Epikutantest-Standardreihe (DKG, Deutsche Kontaktallergie-Gruppe) möglich ( $\triangleright$ Tab. 1).

\section{Interessenkonflikt}

Die Autorinnen/Autoren geben an, dass kein Interessenkonflikt besteht. 
L. Klimek ${ }^{1}$, M. Spielhaupter ${ }^{1}$, A. Alali ${ }^{1}$,

L. Freudelsperger ${ }^{2}$, M. Cichy ${ }^{1}$, T. Huppertz ${ }^{2}$, J. Hagemann²

${ }^{1}$ Zentrum für Rhinologie und Allergologie, Wiesbaden

${ }^{2}$ Hals-Nasen-Ohrenklinik und Poliklinik, Universitätsmedizin Mainz
Korrespondenzadresse

Professor Dr. med. L. Klimek

Zentrum für Rhinologie und Allergologie

Wiesbaden

An den Quellen 10

65183 Wiesbaden

Tel.: ++49/611/3086080

Ludger.Klimek@Allergiezentrum.org

Literatur

[1] Viruses CSGotICoTo. The species Severe acute respiratory syndrome-related coronavirus: classifying 2019-nCoV and naming it SARSCoV-2. Nature microbiology 2020; 5 (4): 536544
[2] Arbeitsmedizin BfAu. Informationen zum rechtmäßigen Inverkehrbringen von PSA in Deutschland 2020.

[3] Arbeitsmedizin BfAu. FAQ: Sonderregelungen für die aktuelle Pandemie-Situation 2020.

[4] Klimek L, Werfel T, Vogelberg C. Weißbuch Allergie in Deutschland; Springer; 2019

[5] Amann PM. „Das kleine $1 \times 1$ der Kontaktallergene“ - Teil 6 Desinfektionsmittel. Allergojournal 2018; 2: 89-93

Bibliografie

DOI https://doi.org/10.1055/a-1189-6301

Online-Publikation: 3.6.2020

Laryngo-Rhino-Otol 2020; 99: 475-477

(c) Georg Thieme Verlag KG, Stuttgart · New York ISSN 0935-8943 